\title{
Neural Substrate of Spatial Memory in the Superior Colliculus after Damage to the Primary Visual Cortex
}

\author{
Kana Takaura, ${ }^{1,2}$ Masatoshi Yoshida, ${ }^{1,2}$ and Tadashi Isa ${ }^{1,2,3}$ \\ ${ }^{1}$ School of Life Science, the Graduate University for Advanced Studies (SOKENDAI), Hayama 203-0193, Japan, ${ }^{2}$ Laboratory of Behavioral Development, \\ Department of Developmental Physiology, National Institute for Physiological Sciences, National Institutes of Natural Sciences, Okazaki 444-8585, Japan, \\ and ${ }^{3}$ Core Research for Evolutional Science and Technology, Japan Science and Technology Agency, Kawaguchi 333-0012, Japan
}

In the primate brain, the primary visual cortex (V1) is a major source of visual information processing in the cerebral cortex, although some patients and monkeys with damage to the V1 show visually guided behaviors in the visual field affected by the damage. Until now, behaviors of the surviving brain regions after damage to V1 and their contribution to the residual visual functions remain unclear. Here, we report that the monkeys with a unilateral lesion of V1 can make not only visually guided saccades but also memory-guided saccades (MGS) into the affected visual field. Furthermore, while the monkeys were performing the MGS task, sustained activity was observed in a large fraction of the neurons in the superior colliculus ipsilateral to the lesion, which has been supposed as a key node for recovery after damage to V1. These neurons maintained the spatial information throughout the delay period regardless of whether they exhibited saccadic bursts or not, which was not the case on the intact side. Error analysis revealed that the sustained activity was correlated with monkeys' behavioral outcome. These results suggest that the ipsilesional SC might function as a neural substrate for spatial memory in the affected visual field. Our findings provide new insight into the understanding of the compensatory mechanisms after damage to V1.

\section{Introduction}

In the primate brain, the primary visual cortex (V1) is a major source of visual information processing in the cerebral cortex, and it is well known that damage to V1 leads to loss of visual awareness in the visual field affected by the damage. However, after a recovery period, some patients and the monkeys with damage to the V1 show visually guided behaviors in the affected visual field, such as localization of a target by hand or eye movements in a forced choice condition (Pöppel et al., 1973; Sanders et al., 1974; Weiskrantz et al., 1974; Cowey and Stoerig, 1995; Moore et al., 1995), which is a phenomenon called "blindsight" (Weiskrantz, 1986).

How the residual abilities are achieved without V1 remains unclear. Residual visual functions are observed after the recovery period for several weeks (Mohler and Wurtz, 1977; Segraves et al., 1987; Cowey and Stoerig, 1995; Yoshida et al., 2008; Ikeda et al., 2011), suggesting a role for plastic changes in the brain after damage to V1. Thus, the surviving brain regions may change their contribution to the process of visuomotor transformation. Actually, studies using diffusion tensor imaging suggest a possibility of

Received Oct. 1, 2010; revised Dec. 20, 2010; accepted Jan. 14, 2011.

This work was supported by Core Research for Evolutionary Science and Technology (CREST) from Japan Science and Technology Agency (JST), Human Frontier Science Program, and Ministry of Education, Culture, Sports, Science, and Technology of Japan Grants-in-Aid for Science Research 17021041, 18200027, and 21500377. We are grateful to Michele A. Basso and her colleague for comments on an earlier version of the manuscript.

Correspondence should be addressed to Dr. Tadashi Isa, National institute for Physiological Sciences, 38 Nishigonaka, Myodaiji, 0kazaki, Aichi, 444-8585, Japan. E-mail: tisa@nips.ac.jp.

K. Takaura's present address: Brain Science Institute, Tamagawa University, Tamagawagakuen 6-1-1, Machida, Tokyo 194-8610, Japan.

DOI:10.1523/JNEUROSCI.5143-10.2011

Copyright $\odot 2011$ the authors $\quad 0270-6474 / 11 / 314233-09 \$ 15.00 / 0$ a large-scale reorganization across the cerebral and subcortical structures in response to the damage to V1 (Leh et al., 2006; Bridge et al., 2008). One of the subcortical structures reported to have atypical connectivity in patients with blindsight is the superior colliculus (SC) (Leh et al., 2006), and the involvement of the $\mathrm{SC}$ in residual visual functions is also supported by a previous study examining the effect of a combined lesion of V1 and the SC (Mohler and Wurtz, 1977). Although the SC is known to work as a center of the visuomotor transformation in conjunction with the cerebral regions in the intact brain (Wurtz et al., 2001; Boehnke and Munoz, 2008), the changes in the functional role of the SC through the recovery process after damage to $\mathrm{V} 1$ remain unclear.

In the present study, we report that monkeys with a unilateral lesion of V1 can make not only visually guided saccades (VGS) but also memory-guided saccades (MGS) into the affected visual field, and a large fraction of the neurons in the SC ipsilateral to the lesion (ipsi SC) exhibit sustained activity throughout the retention interval of the MGS task. We recorded neuronal activity from the ipsi SC while the monkeys were performing the VGS or MGS tasks in the affected visual field and compared this activity with that in the SC contralateral to the lesion (contra SC) as a control. The results suggest a possibility that the ipsi SC serves not only as a center of visuomotor transformation but also as a neural substrate of spatial memory in the affected visual field.

\section{Materials and Methods}

Experimental procedures. All the experimental procedures were performed in accordance with the Guidelines for Proper Conduct of Animal Experiments of the Science Council of Japan and approved by the Committee for Animal Experiment at the National Institutes of Natural Sciences. Two Japanese monkeys (Macaca fuscata; monkey T, female, body 
A

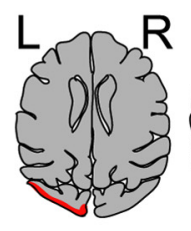

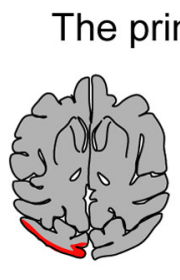

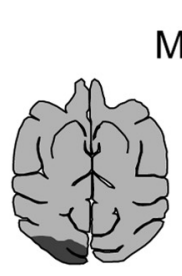

Monk A, post-op
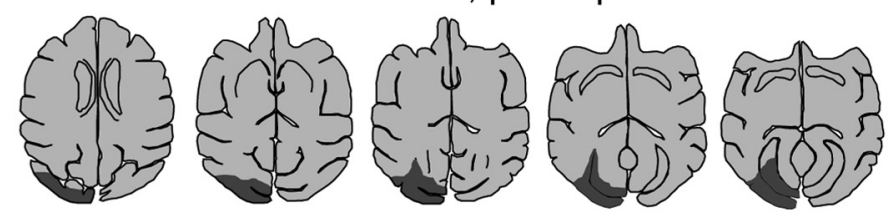

Monk T, post-op
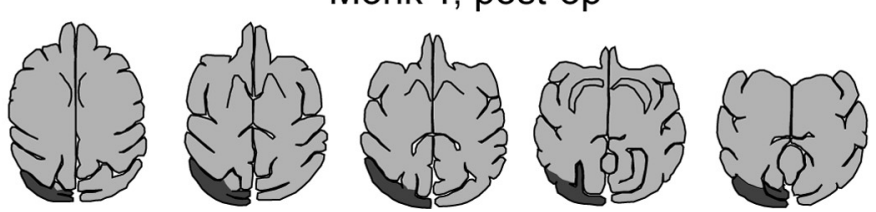

B
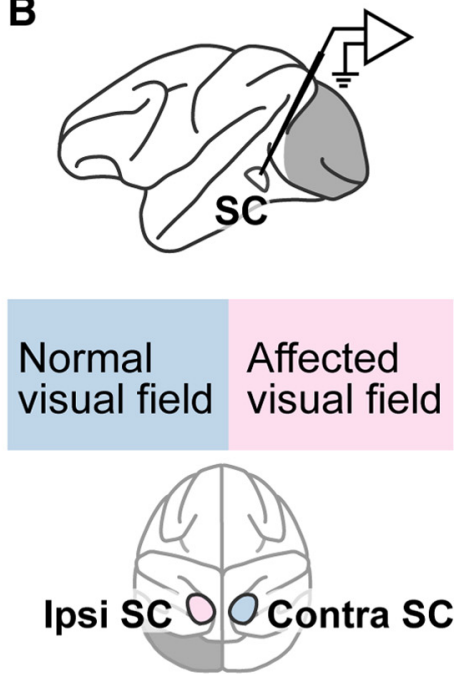

Figure 1. V1 and the extent of the lesion $(\boldsymbol{A})$ and the experimental conditions $(\boldsymbol{B})$. $\boldsymbol{A}$, Top, The figures are line images traced from axial slices of MR images acquired at 1 week before the lesion. $\mathrm{V} 1$ is denoted in red (monkey A). The estimated lesion site (depicted in dark gray) is overlaid on the line trace of the axial slices of the MR images acquired after the lesion in monkey $A$ (middle) and in monkey T (bottom). B, Schema of the experimental conditions. In both monkeys, the left V1 was removed. Thus, the right hemifield was affected and the left visual field was normal.

weight of $6.5 \mathrm{~kg}$; monkey A, male, body weight of $9.0 \mathrm{~kg}$ ) were implanted with head holders and scleral search coils to measure eye movements. After training on the saccade tasks, including the VGS and MGS tasks (described below, The VGS task and the MGS task), the left V1 was removed by suction and a recording chamber was implanted. The data in this study were acquired after the monkeys' performance in the VGS task reached a steady state after the V1 lesion.

V1 lesion. The procedures used to examine the extent of the lesion, details of the postoperative training, and recovery processes were described in our previous study using the same subjects as the current study (Yoshida et al., 2008). Briefly, we examined the extent of the lesion anatomically and functionally. To examine the extent of the lesion anatomically, magnetic resonance imaging (MRI) was performed preoperatively and postoperatively. We confirmed that almost all of the intended regions (the posterior half of the operculum, dorsal and ventral leaves and roof of the calcarine sulcus, and the most posterior part of the stem of the calcarine sulcus) were removed (Fig. 1A). Based on published reports (Daniel and Whitteridge, 1961; Van Essen et al., 1984), we concluded that at least the region corresponding to the visual field from $5^{\circ}$ to $25^{\circ}$ in eccentricity was removed. Because we intended to remove V1 as completely as possible, the lesion may include parts of the extrastriate areas such as V2 or V3. To examine the extent of the lesion functionally, we measured the sensitivity to luminance contrast in each visual field separately. In the VGS task (step condition), the luminance contrast of the target was systematically varied from 0.05 to 0.9 . The target was presented at a position $5^{\circ}$ to $25^{\circ}$ in eccentricity and from $-60^{\circ}$ to $60^{\circ}$ from the horizontal line in a direction separated by $30^{\circ}$. At each position, the success rates were calculated and the psychometric function was drawn. In this process, trials were judged as correct if the direction of the saccade was within $\pm 15^{\circ}$ from the direction of the targets. The threshold for the luminance contrast at each position was evaluated from each psychometric function. The thresholds in the affected visual field were consistently higher than those in the normal visual field. Thus, we confirmed that the visual field corresponding to the lesion anatomically estimated by the postoperative MRI was functionally affected. In our previous study, we also examined the possibility that the monkeys used light scattering of the visual stimuli presented in the affected visual field (Campion et al., 1983; Gross et al., 2004). We examined whether they could respond to a $1.52^{\circ}$ visual stimuli (the maximal size of visual stimuli used in the current study) presented in the blind spot in one eye, and we found that they could not make saccades to the target presented in the blind spot. Thus, it is unlikely that the monkeys used light scattering when they made saccades toward the visual stimuli presented in the affected visual field.

The VGS task and the MGS task. While the monkeys were fixating on the fixation point (FP) (800-1200 ms in monkey T, 500-900 ms in monkey A), another visual stimulus was presented in the peripheral visual field. In the VGS task, the stimulus ("target") remained on the display. After a short delay (fixed at $500 \mathrm{~ms}$ ), the FP disappeared (see Fig. 2 A, left). The monkeys were required to make a saccade toward the target. In the MGS task, the stimulus ("cue") transiently flashed (100 ms) and disappeared. The offset of the cue was followed by a temporal interval ("delay period") of 800-2400 ms (randomized). After that, the FP disappeared (see Fig. $2 B$, left). The monkeys were required to make a saccade toward the position of the cue, based on their memory. The following parameters were common to the VGS and MGS tasks. The trials were judged as correct if the saccade landed within the invisible window of a circle with a $5^{\circ}$ to $15^{\circ}$ radius. The radius of the window was adjusted based on the eccentricity of the targets and the cues. In the majority of the sessions, the size of the stimuli was set at a diameter of $0.91^{\circ}$ or $1.52^{\circ}$ in monkey $\mathrm{T}$ and monkey A, respectively. In some sessions, the size of the stimuli was set at $0.42^{\circ}$ to collect a sufficient number of error trials. Background luminance was set at $1 \mathrm{~cd} / \mathrm{m}^{2}$, and the luminance contrast of the visual stimuli was 0.9 .

Recording procedures. Epoxylite-coated tungsten microelectrodes (impedance: 1-3 $\mathrm{M} \Omega$ at $1 \mathrm{kHz}$ ) were used. After isolation of a single neuron, we first investigated its receptive field (RF) to decide the two positions for presentation of the targets or the cues within the visual field contralateral to the recording site. One was at the putative center of the $\mathrm{RF}\left(\mathrm{RF}_{\mathrm{in}}\right)$, and the other was separated by $90^{\circ}$ from the first position $\left(\mathrm{RF}_{\text {out }}\right)$ (see Fig. $3 A, B$, top). In one neuron in the ipsi SC, the RF of which was centered on the horizontal line, $\mathrm{RF}_{\text {in }}$ and $\mathrm{RF}_{\text {out }}$ were separated by $75^{\circ}$. The $\mathrm{RF}$ was explored with the VGS task or with presentation of visual stimuli, while the monkeys were fixating on the FP. Subsequently, activity during the VGS and MGS tasks was recorded in different blocks. In each neuron, activity was recorded at least five trials for both the VGS and MGS tasks, respectively.

Data analysis: classification of the neurons. In the offline analysis, trials were judged as correct if the direction of saccade was within $45^{\circ}$ from the direction of the targets or the cues. We characterized the neurons based 
A Visually guided delayed saccade task
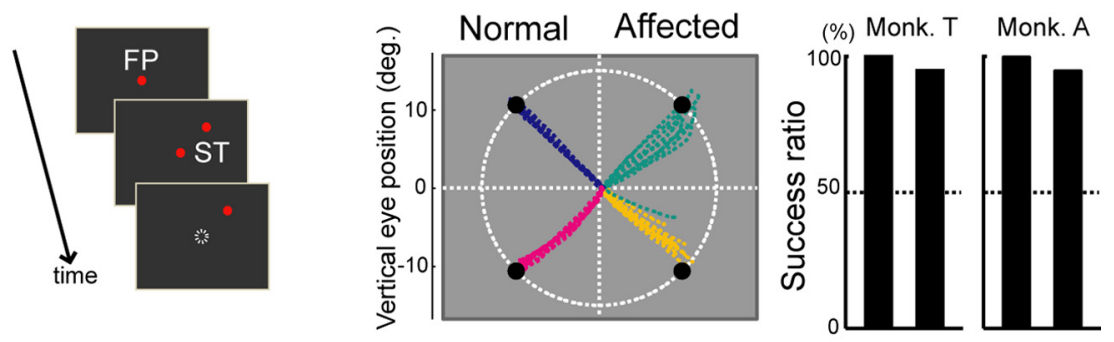

\section{B Memory-guided saccade task}
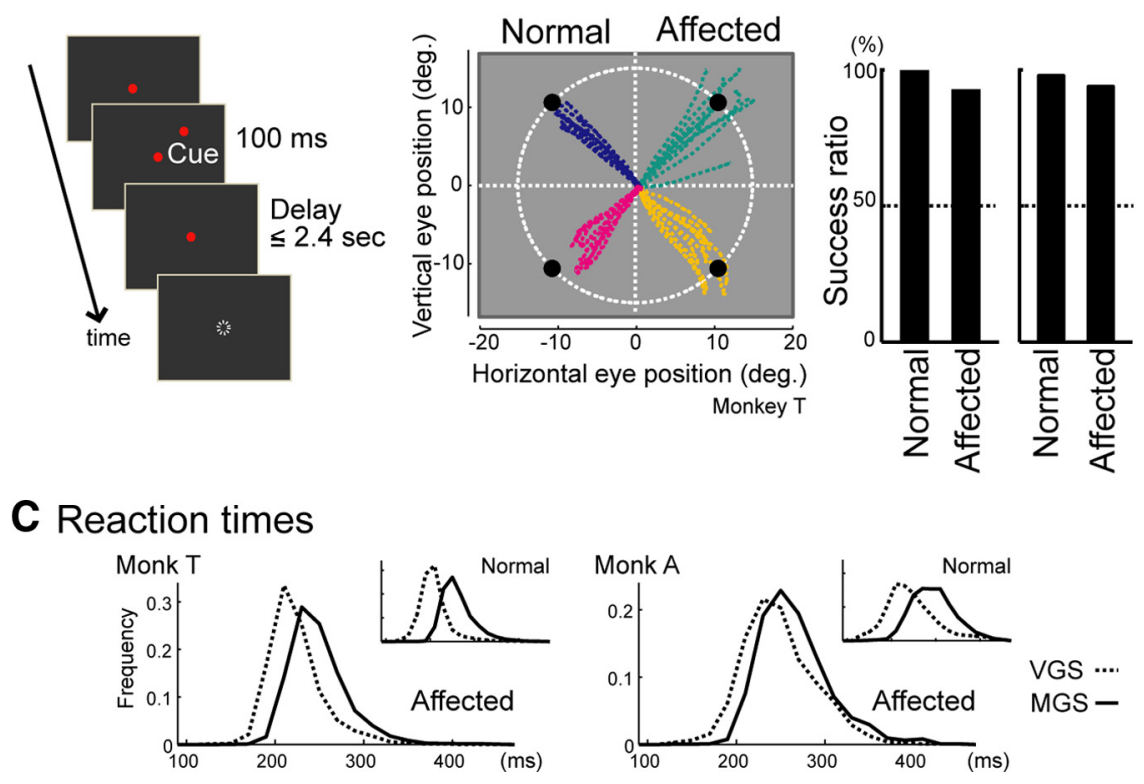

Figure 2. Behavioral results for the VGS and MGS tasks. $A, B$, Left, Task schema; middle, trajectories; right; the success rates for each visual field in the two monkeys. In the middle panel, the differences in color indicate differences in the position of the target or the cue. ST, Saccade target. C, Distributions of the saccadic reaction times in monkey T (left) and monkey A (right). The dashed lines represent the results in the VGS task, while the solid lines represent the results in the MGS task. The insets to the right and above show the distribution of the saccadic reaction times in the normal visual field.

on the firing properties during the VGS task. First, we produced the mean spike density function aligned to the target onset or saccade onset with a Gaussian kernel ( $\sigma=4 \mathrm{~ms})$ in each neuron. To examine whether the neuron exhibited a significant response to the visual stimuli presented inside the RF, a peak in the averaged spike density function was detected in the $100 \mathrm{~ms}$ epoch from 50 to $150 \mathrm{~ms}$ after the target onset, and the mean firing rates were calculated within the $50 \mathrm{~ms}$ epoch centered on the time of the peak (from $25 \mathrm{~ms}$ before to $25 \mathrm{~ms}$ after the peak). The firing rates were compared to those in the baseline epoch starting from $300 \mathrm{~ms}$ before and ending at the time of the target onset. If the statistical comparison was significant (one-tailed paired $t$ test, $\alpha=0.05$ ), the neuron was considered to have visual responses. The mean firing rate during the $50 \mathrm{~ms}$ interval was taken as the magnitude of the visual response. The latency of the visual response was defined as the time point at which the poststimulus activity (activity over $40 \mathrm{~ms}$ after the stimulus onset) exceeded 2 SDs of the baseline activity (in the $100 \mathrm{~ms}$ epoch starting from 60 $\mathrm{ms}$ before the stimulus onset). Neurons without visual responses were excluded from subsequent analyses. The neurons with visual responses were classified into two groups: visuomotor neurons and visual neurons. The neurons with both visual responses and saccadic bursts were classified as visuomotor neurons, and neurons with only visual responses were classified as visual neurons. The presence or absence of saccadic bursts was determined based on activity aligned to the saccade onset. The mean firing rates were calculated within the $50 \mathrm{~ms}$ epoch centered on the time of the peak in the spike density function, which was detected in the 100 ms epoch centered on the saccade onset. The rates were compared to those in the baseline epoch of 100 ms centered on the FP offset. The mean firing rate during the $50 \mathrm{~ms}$ interval was taken as the magnitude of the saccadic burst. The magnitude of the prestimulus activity was defined as the mean firing rates in the $100 \mathrm{~ms}$ epoch just before the stimulus onset.

Data analysis: evaluation of spatial information during the delay period. To evaluate spatial information during the delay period, the time course of the area under the curve (AUC) of the receiver operating characteristics curve (ROC) was calculated in each neuron by sliding the $100 \mathrm{~ms}$ time window by $10 \mathrm{~ms}$. In each time window, the mean firing rates in the $\mathrm{RF}_{\text {in }}$ trials were compared with those in the $\mathrm{RF}_{\text {out }}$ trials; the ROC was then drawn and the AUC was calculated. The AUC value indicates the probability that an ideal observer can discriminate between the $\mathrm{RF}_{\text {in }}$ and $\mathrm{RF}_{\text {out }}$ trials based on neuronal activity. An AUC of 1 indicates that the observer can perfectly discriminate between the two conditions and that the mean firing rates in the $\mathrm{RF}_{\text {in }}$ trials are higher than those in the $\mathrm{RF}_{\text {out }}$ trials. An AUC of 0.5 indicates that the observer performed at the chance level and that the mean firing rates are the same in the $\mathrm{RF}_{\text {in }}$ and $\mathrm{RF}_{\text {out }}$ trials.

Data analysis: error analysis. In the error analysis, neurons with at least three error trials in the $\mathrm{RF}_{\text {in }}$ trials or at least three error trials in the $\mathrm{RF}_{\text {out }}$ trials were extracted. The mean firing rates during the $300 \mathrm{~ms}$ interval just before the FP offset were calculated for each condition. We confirmed that the monkeys' performance in the recording block of each neuron was above chance level (binomial test, $\alpha=0.05$ ).

\section{Results}

Monkeys with a unilateral V1 lesion

The unilateral V1 was removed by suction in two Japanese monkeys (monkey $\mathrm{T}$, female, and monkey A, male) (Fig. $1 A$ ) that had been trained on the VGS task (Fig. 2A, left) and the MGS task (Fig. $2 B$, left). The recovery process and the procedures to examine the extent of the lesion were described in our previous study (Yoshida et al., 2008). Briefly, the extent of the lesion was confirmed anatomically by the postoperative MRIs and behaviorally by the sensitivity-to-luminance contrast (see Materials and Methods). Based on these results, we concluded that almost all of the left V1 had been removed. Thus, the right visual hemifield was referred to as the affected visual field (Fig. $1 B$, bottom).

\section{Performance on the VGS and the MGS tasks after lesion of the V1}

Behavioral testing and the recording of the neuronal activity from the SC began 3.5 to 4 years after lesion of the V1, and the data from the VGS and MGS task presented here were acquired in the same experimental sessions (107 sessions in monkey T, 49 sessions in monkey A). In Figure 2, $A-C$, the behavioral results in the VGS and MGS tasks are summarized. In the VGS task, the monkeys showed success rates of $94.6 \%(n=3438 / 3635)$ in monkey $\mathrm{T}$ and $94.6 \%(n=1736 / 1836)$ in monkey A (Fig. $2 A$, right), and these results were significantly above chance level (binomial test, $p<0.0001$ in both monkeys). The averaged saccadic reaction 
times were 185.3 and $194.9 \mathrm{~ms}$, respectively, in each monkey (Fig. 2C). In the MGS task, the success rates were $92.2 \%$ $(n=7375 / 8002)$ in monkey $\mathrm{T}$ and $93.8 \%$ $(n=2386 / 2544)$ in monkey A (Fig. 2B, right), and these results were also significantly above chance level (binomial test, $p<0.0001$ in both monkeys). The averaged saccadic reaction times were 210.0 and $212.8 \mathrm{~ms}$ (Fig. 2C), which were significantly longer than those in the VGS task (Mann-Whitney $U$ test, $p<10^{-13}$ in both monkeys) as observed in the normal visual field or reported in previous studies with naive monkeys (Funahashi et al., 1989). These results confirmed that there was residual visual function after the V1 lesion.

\section{Single unit-recording form the SC}

In total, the activity of 72 neurons in the ipsi SC (47 neurons in monkey $\mathrm{T}$ and 25 neurons in monkey A) was recorded while the monkeys were performing the tasks in the affected visual field (Fig. $3 A$, top). As a control, the activity of 70 neurons in the SC contralateral to the lesion of the same monkeys (42 neurons in monkey $\mathrm{T}$ and 28 neurons in monkey A) was recorded while the monkeys were performing the tasks in the normal visual field (Fig. $3 B$, top). The neurons with visual responses ( 58 neurons in the ipsi SC and 66 neurons in the contra $\mathrm{SC})$ were investigated in the following analysis. The depths of the recording sites from the surface of the SC ranged from 310 to $2389 \mu \mathrm{m}$ in the ipsi SC and from 150 to $3050 \mu \mathrm{m}$ in the contra SC. Distributions of the depth of recording sites and maps of recording location are shown in Figure 3, $A$ and $B$ (bottom). The surface of the $\mathrm{SC}$ was identified by the change of the background noise as the electrodes were penetrating from the recording chamber that was tilted $40^{\circ}$ posterior to the vertical axis. The mean depths of the recording sites were not significantly different between the ipsi and contra SCs (two-tailed $t$ test, $p>0.4$ ), and no significant difference in their distribution was observed (Kolmogrov-Smirnov test, $p>0.5$ ). In both the ipsi and the contra SCs, neuronal activities were recorded primarily from the deeper layer of the SC. A few neurons (two in the ipsi SC and two in the contra SC) may have been recorded in the superficial layer of the SC and were recorded within $500 \mu \mathrm{m}$ from the putative surface of the SC ( 310 and $385 \mu \mathrm{m}$ in the ipsi SC, 150 and $230 \mu \mathrm{m}$ in the contra SC). Because their activity did not appear to differ from that of the other neurons located in the deeper layer, they were merged in the following analysis.

\section{Neuronal activities in the SC during the VGS task}

Neurons with visual responses were classified into visuomotor neurons and visual neurons, based on the presence or absence of saccadic bursts (See Materials and Methods). The activity of 46 visuomotor neurons and 12 visual neurons was recorded from the ipsi SC, and the activity of 50 visuomotor neurons and 16 visual neurons was recorded from the contra SC. Neuronal activity during the VGS task is shown in Figure 4 (Fig. $4 A$, representative neurons; Fig. $4 B, C$, population). The targets were presented at either of two positions: inside the RF or outside the

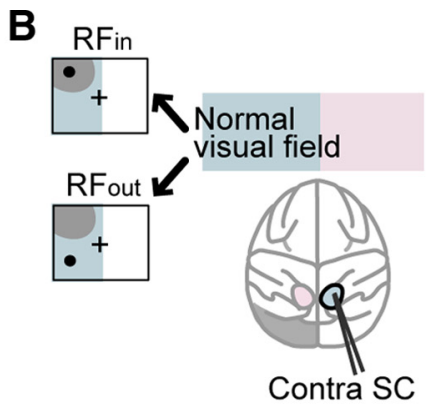

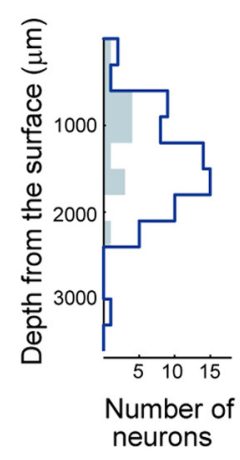

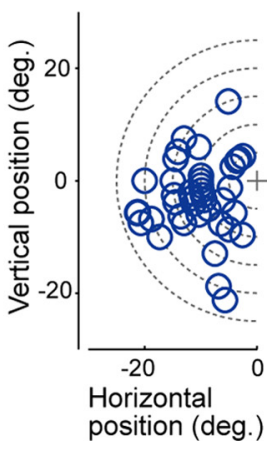

position (deg.)
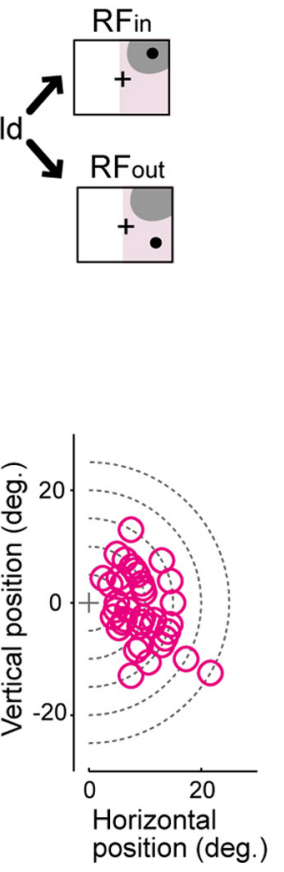

Figure 3. Recording from the ipsi and contra $S C S . A, B$, Recording configuration (top row), a distribution of the depth of recording sites top). The target or the cue was presented inside the $\mathrm{RF}, \mathrm{RF}_{\mathrm{in}}$, or outside the $\mathrm{RF}, \mathrm{RF}_{\text {out }}$, separated by $90^{\circ}$ in direction. In the lower left panels, the open histograms indicate the visuomotor neurons and the shaded histograms indicate the visual neurons.

RF (Fig. 3). Here we called the trials with the target presented inside the RF as " $\mathrm{RF}_{\text {in }}$ trials" and those with the target presented outside the RF as "RF $F_{\text {out }}$ trials" (see Material and Methods). To evaluate the spatial information carried by these neurons, we compared the instantaneous firing rates between the $\mathrm{RF}_{\text {in }}$ and $\mathrm{RF}_{\text {out }}$ trials in each neuron and calculated the area under the curve (AUC) of the receiver operating characteristics curve (See Materials and Methods). The time course of the AUC was computed for each neuron and averaged respectively in the ipsi and contra SCs (Fig. 4B, C, bottom rows) (see Materials and Methods). After the peak, the AUC declined more slowly in the ipsi SC than in the contra SC, but it fell to almost the same level as in the contra SC until the FP offset. During the $100 \mathrm{~ms}$ epoch just before the FP offset, the AUCs did not differ significantly between the ipsi and contra SCs (Mann-Whitney $U$ test, $p>0.6$ ).

The gross magnitudes of the neuronal activities during the VGS task were similar between the ipsi SC and the contra SC with respect to population activity (Fig. $5 A$, left, and $5 B$ ). The magnitudes of the visual responses were not significantly different between the ipsi SC and contra SC for either visual or visuomotor neurons (Mann-Whitney $U$ test, $p>0.05$ ) (Fig. 5A, left column). In the ipsi SC, the average firing rates of the visuomotor and visual neurons were 69.8 and 65.9 spikes/s, respectively, and those in the contra SC were 75.3 and 81.3 spikes/s, respectively. The average latencies of visual response were 59.0 and $54.0 \mathrm{~ms}$ in the ipsi SC in the visuomotor and visual neurons, respectively, while they were 43.4 and $47.2 \mathrm{~ms}$, respectively, in the contra SC (Fig. 5A, right column). Thus, in the visuomotor neurons, the latencies were significantly different between the ipsi and contra SCs (Mann-Whitney $U$ test, $p<0.001$ ). The magnitudes of the saccadic bursts of visuomotor neurons were not significantly different between the ipsi SC and contra SC (Fig. 5B). The mean 


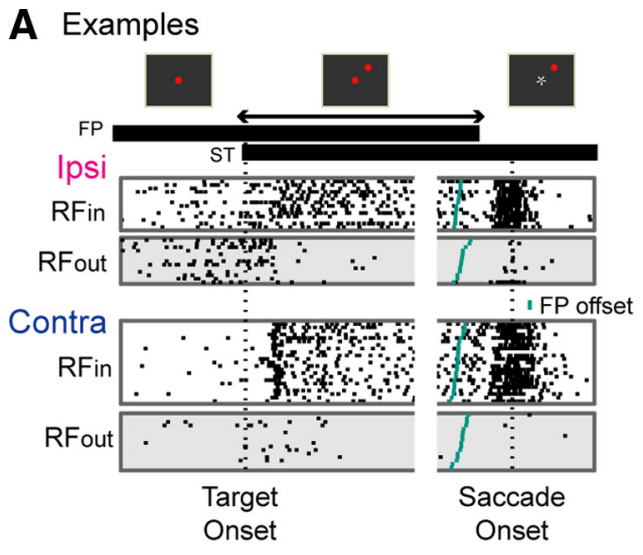

B Population activity of visuomotor neurons

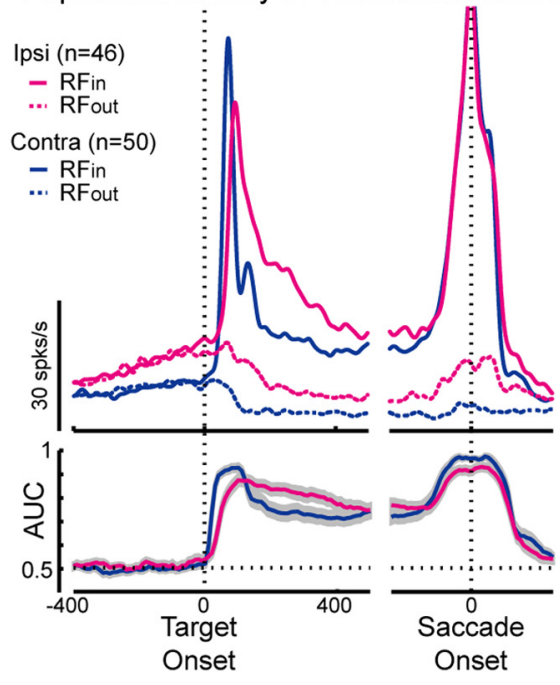

C Population activity of visual neurons

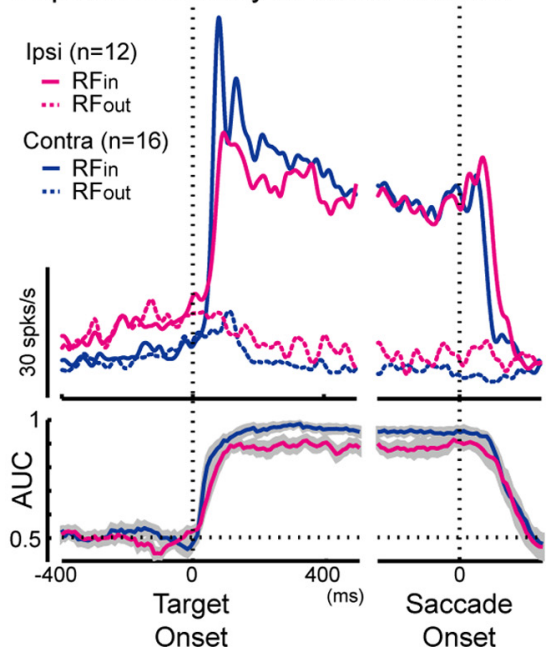

Figure 4. Neuronal activity during the VGS task. A, Schema of the task and the raster plots of representative neurons in the ipsi SC (top row) and the contra SC (bottom row). ST, Saccade target. $\boldsymbol{B}, \boldsymbol{C}$, Activity of visuomotor neuron and visual neuron populations. Top rows, Spike density functions; bottom rows, time course of the AUC (area under the curve of the receiver operating characteristics curve, see Material and Methods). The left column shows the activity aligned with the target onset, and the right column shows the activity aligned with the saccade onset.
A Visual response
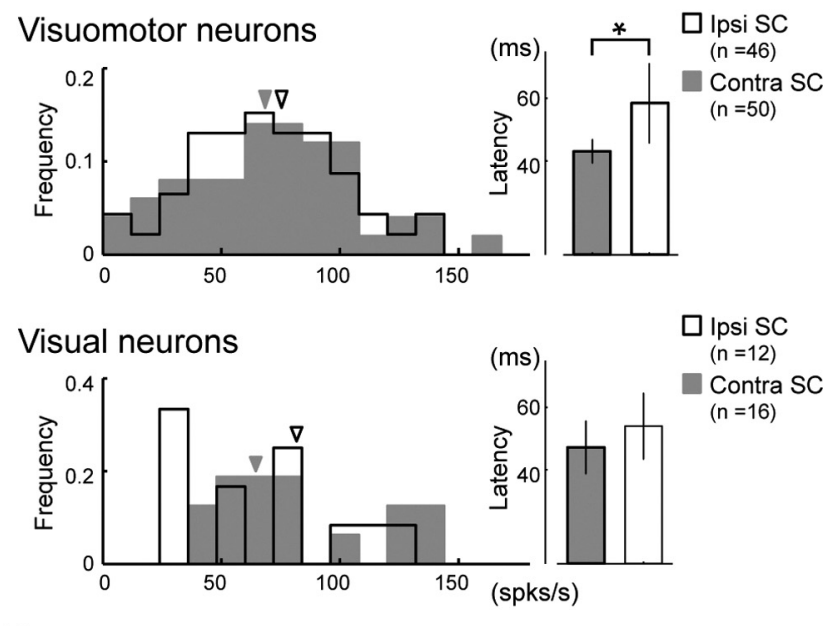

B Saccadic burst Visuomotor neurons

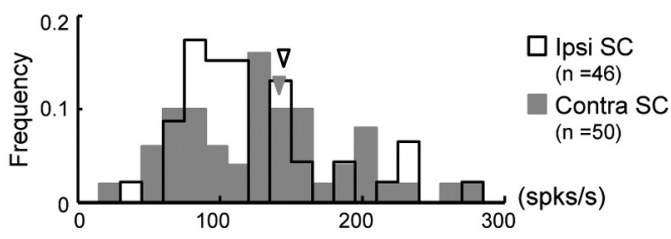

C Prestimulus activity
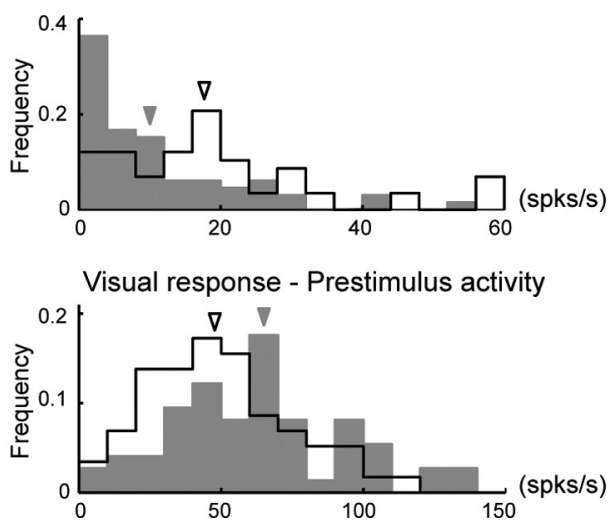

$\square$ Ipsi SC $(n=58)$

Contra SC $(n=66)$

Figure 5. Comparison of the basic properties of visual response and saccadic burst between the ipsi and the contra SCs. A, Comparison of visual responses. Right column, Magnitudes; left column, latencies. Top, Visuomotor neurons; bottom, visual neurons. Error bars in the bar graphs indicate SD. B, Comparison of the magnitude of the saccadic bursts. C, Top, Comparison of the magnitudes of the prestimulus activity. Bottom, Distribution of the net magnitudes of visual responses calculated by subtraction of the prestimulus activity from the gross magnitudes of visual response. $\boldsymbol{A}-\boldsymbol{C}$, The open histograms indicate the ipsi $\mathbf{S C}$, and the shaded histograms indicate the contra SC. The arrowheads indicate the averaged firing rates; spks/s, Spikes per second.

firing rates were $144.6 \mathrm{spikes} / \mathrm{s}$ in the ipsi SC and $140.5 \mathrm{spikes} / \mathrm{s}$ in the contra SC (Mann-Whitney $U$ test, $p>0.05$ ).

In addition to the visual response and the saccadic burst, we also found the increase of firing rates before the target onset in the ipsi SC. The magnitudes of the prestimulus activity were 19.3 spikes/s in the ipsi SC and 11.3 spikes/s in the contra SC (Fig. 5C, top), and these values were significantly different (Mann-Whitney $U$ test, $p<$ 0.001 ). When we calculated the net magnitudes of visual responses by subtraction of the prestimulus activity, the magnitudes of the visual responses between the ipsi and contra SCs were 
significantly different (49.7 spikes/s and 65.4 spikes/s respectively, Mann-Whitney $U$ test, $p<0.01$ ).

\section{Neuronal activities in the SC during the MGS task}

A striking difference in neuronal activity between the ipsi and contra SCs was observed during the retention interval in the MGS task. The activity of representative neurons in both the ipsi and contra SCs in correct trials is shown as raster plots in Figure 6A. The representative neuron in the ipsi SC (Fig. 6A, top) maintained firing from just after the presentation of the cue until the FP offset ("delay period"), when the cue was presented inside the $\mathrm{RF}$ ( $\mathrm{RF}_{\text {in }}$ trials). When the cue was presented outside the $R F\left(\mathrm{RF}_{\text {out }}\right.$ trials), the neuron kept silent during this period. In contrast, the representative neuron in the contra SC (Fig. 6A, bottom) became silent after the visual responses to the cue and then slightly increased its firing rates toward the FP offset in the $\mathrm{RF}_{\text {in }}$ trials. The population activities of the visuomotor neurons and those of the visual neurons are shown in Figure 6, $B$ and $C$, top rows. Similar to the representative neuron, the majority of the neurons in the ipsi SC maintained higher firing rates in the $\mathrm{RF}_{\mathrm{in}}$ trials than in the $\mathrm{RF}_{\text {out }}$ trials during the delay period, regardless of whether the neurons exhibited saccadic bursts (Fig. $6 B$ ) or not (Fig. 6C). The time courses of the AUCs are shown in Figure $6, B$ and $C$, bottom rows.

The difference between the temporal profiles of the spatial information maintained in the ipsi SC during the delay period and those in the contra SC is demonstrated by plotting the cumulative distribution of the AUC in epochs 1-3 (Figs. 7, 6B,C) (see Materials and Methods). When the criteria were set at 0.76 $\left(d^{\prime}=1\right), 74.1 \%$ of the neurons in the ipsi SC discriminated the position of the cue in the early part of the delay period (Fig. $7 A$, epoch 2). After epoch 2, a large fraction of the neurons in the ipsi SC maintained the spatial information until the FP offset. In the last part of the delay period (epoch 3), $41.4 \%$ of the neurons discriminated the position of the cue. On the other hand, in the majority of the neurons of the contra SC, the spatial information was lost just after epoch 1 . Even during the early part of the delay period (epoch 2), only $9.1 \%$ of the neurons discriminated the $\mathrm{RF}_{\mathrm{in}}$ and $\mathrm{RF}_{\text {out }}$ trials (Fig. $7 B$ ). Although the AUC in the contra SC was significantly higher than in the ipsi SC (two-tailed Mann-Whitney $U$ test, $p<0.001$ ) in epoch 1 , the relationship reversed (two-tailed Mann-Whitney $U$ test, $p<$ 0.001 ) in epoch 2 and epoch 3 . These results demonstrated that a high proportion of the neurons in the ipsi SC maintained spatial information regarding the cue throughout the delay period, unlike the neurons in the contra SC. epochs $1-3$.

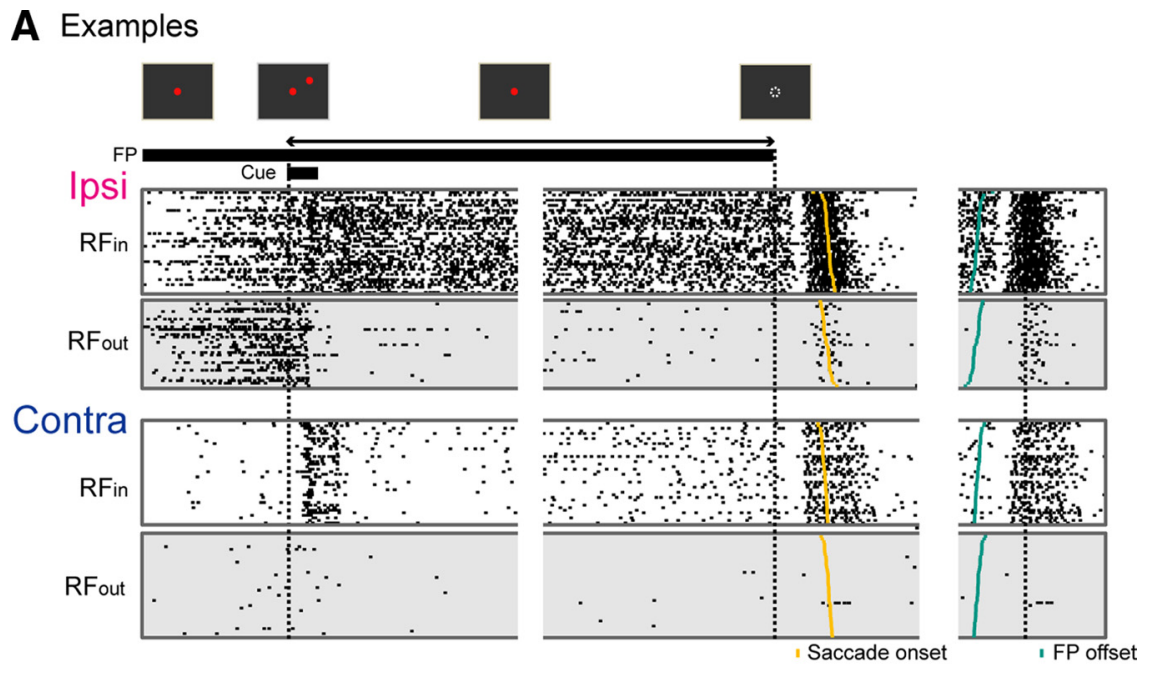

B Population activity of visuomotor neurons
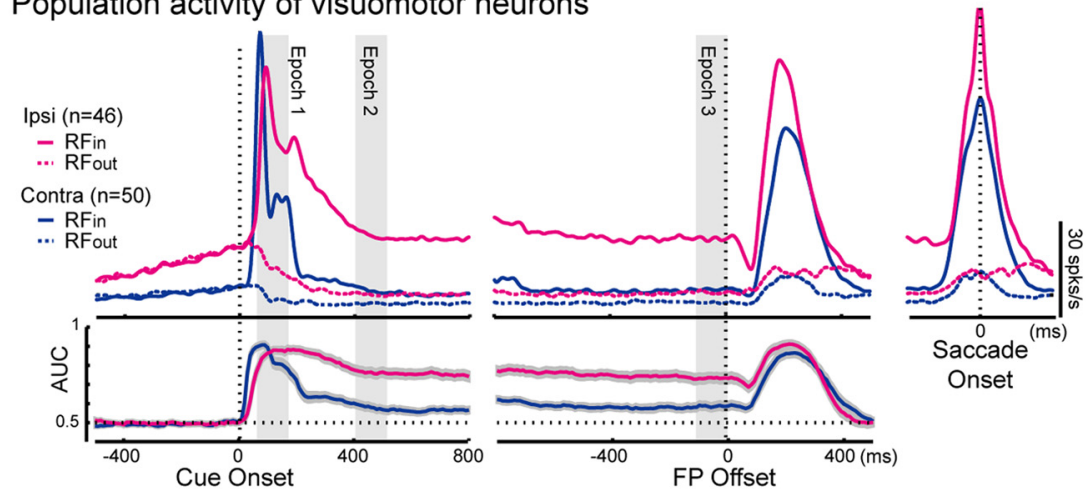

\section{Population activity of visual neurons}
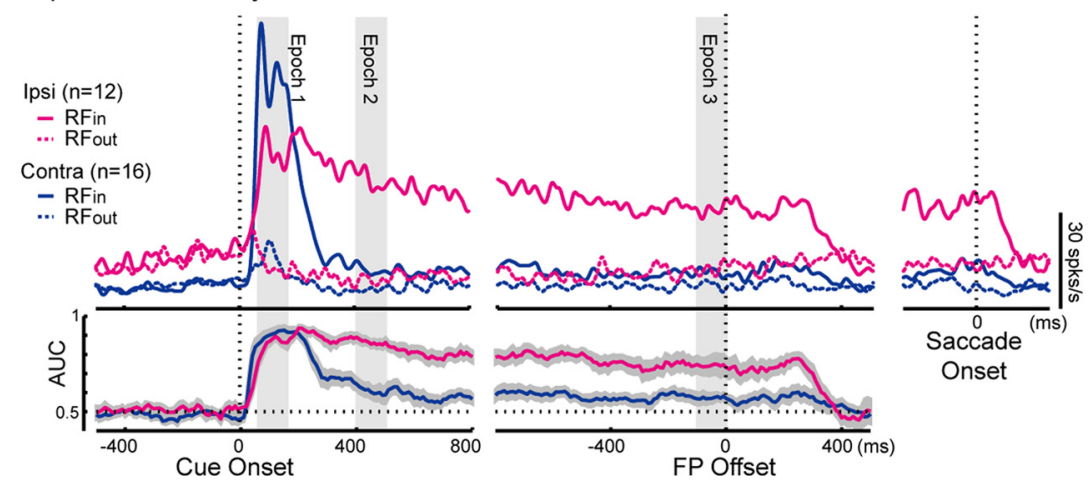

Figure 6. Neuronal activity during the MGS task. $A$, Schema of the task and the raster plots of representative neurons in the ipsi SC (top row) and contra SC (lower row). B, C, Activity of visuomotor neuron and visual neuron populations. Top rows, Spike density functions; lower rows, time course of the AUC. The left columns show activity aligned with the cue onset, the middle columns show activity aligned with the FP offset, and the right columns show activity aligned with the saccade onsets. Shaded areas indicate

Analysis of the error trials revealed that neuronal activity in the ipsi SC during the delay period was correlated with the monkeys' behavioral outcomes (Fig. 8). In the $\mathrm{RF}_{\text {in }}$ trials, when the monkeys made error saccades toward the locations outside the RF ("type I error"), neuronal activity decayed before the FP offset (Fig. $8 \mathrm{~A}$ ). In contrast, in the $\mathrm{RF}_{\text {out }}$ trials, when the monkeys made error saccades toward the locations inside the RF ("type II error"), the neuron discharged during the delay period (Fig. $8 B$ ). In the population analysis, neuronal activity in the later part of the delay period was significantly lower in the type I error trials than in the correct $\mathrm{RF}_{\text {in }}$ trials, and they were significantly higher in the 


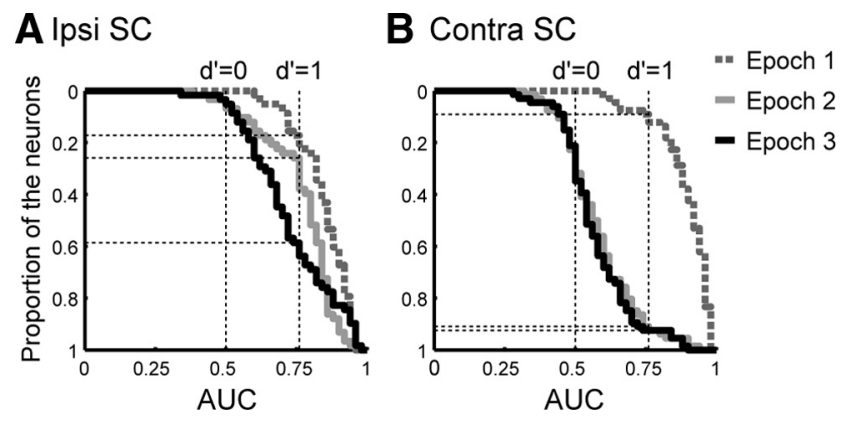

Figure 7. Distribution of the AUC in epochs 1-3 (Fig. $6 B, C$, indicated by shaded areas). $A, B$, The visuomotor and visual neurons were merged in the ipsi SC $(\boldsymbol{A})$ and the contra SC $(\boldsymbol{B})$, respectively. type II error trials than in the correct $\mathrm{RF}_{\text {out }}$ trials (Friedman test, $p<0.0001$; Mann-Whitney $U$ test with Bonferroni's correction for multiple comparisons, $p<0.05$ ) (Fig. $8 C$ ). Thus, the spatial information maintained in the ipsi SC is likely to be used in the processes that determine the monkeys' behavior.

\section{Discussion}

Residual visual functions after damage to $\mathrm{V} 1$ have been reported in some patients and in monkeys with damage to V1 (Pöppel et al., 1973; Sanders et al., 1974; Weiskrantz et al., 1974; Cowey and Stoerig, 1995; Moore et al., 1995; Yoshida et al., 2008; Ikeda et al., 2011), although the neural mechanisms responsible for these abilities are unclear. In the present study, we first demonstrated that monkeys with a unilateral lesion of V1 were able to make not only visually guided saccades but also memory-guided saccades into the affected visual field, and we examined single-unit activity in the ipsi SC while the monkeys were performing the VGS and MGS tasks in the affected visual field. The activity in the ipsi $\mathrm{SC}$ was compared to those in the contra SC while the monkeys were performing the tasks in the normal visual field. Despite the fact that the distribution of the depth of recording sites and the activity during the VGS task were matched between the ipsi and the contra SC, the clear difference between the ipsi and contra SC was observed during the retention interval of the MGS task. A large fraction of the neurons in the ipsi SC exhibited sustained activity in the trials with the cues presented inside the RF and maintained spatial information throughout the delay period. Error analysis revealed that the sustained activity in the ipsi SC was correlated with the monkeys' behavioral outcome. These results suggest that $\mathrm{SC}$ can serve as a neural substrate of spatial memory in the affected visual field, at least for saccades.

Spatial memory can be represented in two ways: retrospective coding and prospective coding (Quintana and Fuster, 1999; Rainer et al., 1999; Curtis and D'Esposito, 2003). Retrospective coding is the memory of past sensory events, and prospective coding is the memory of the future actions. The results of the error analysis showed that activity in the ipsi SC was higher in correct $\mathrm{RF}_{\text {in }}$ trials and type II error trials than in correct $\mathrm{RF}_{\text {out }}$ trials and type I error trials (Fig. 8). That is, activity in the ipsi SC was correlated with the monkeys' saccade direction rather than the actual cue position. These results indicate that prospective information is represented in the ipsi SC. On the other hand, we observed sustained activity not only in the visuomotor neurons but also in the visual neurons. Since visual neurons are thought to be not directly involved in saccade execution, their sustained activity would not represent the prospective information or motor preparation. Rather,
Figure 8. Error analysis. $\boldsymbol{A}, \boldsymbol{B}$, Activity of a representative neuron in the $\mathrm{RF}_{\text {in }} \operatorname{trials}(\boldsymbol{A})$ and $\mathrm{RF}_{\text {out }}$ trials $(\boldsymbol{B})$. In this session, we used a smaller sized cue to collect a sufficient number of error trials. C, Population analysis of the error trials. Activity during the $300 \mathrm{~ms}$ of the last part of the delay period was compared between the correct trials and error trials. The visuomotor and visual neurons were merged. Data represent mean $\pm \mathrm{SE} ;{ }^{*} p<0.05$ according to Mann-Whitney $U$ test with Bonferroni's correction for multiple comparison; spks/s, Spikes per second. 
the sustained activity of visual neurons would represent an intermediary process between visual inputs and motor outputs. Thus, the activity in the ipsi SC bridging the temporal gap between sensory information and motor execution would represent not only the prospective information but also the processes of the visuomotor transformation preceding the decision of saccade direction. However, involvement of SC activity in the visuomotor processing for other task contexts such as hand manipulation remains to be tested in future.

Involvement of the SC in residual visual functions has been a controversial issue in studies about the neural mechanism of residual visual functions after damage to the V1 (Dineen et al., 1982; Rodman et al., 1989; Morris et al., 1999, 2001; Ro et al., 2004; Boyer et al., 2005; Isa and Yoshida, 2009; Leh et al., 2010; Schmid et al., 2010); in most of these studies the point of the argument concerns the role of the SC as a component of the visual pathways bypassing V1. By showing that the SC is involved in spatial memory, our results suggest that the SC contributes to the recovery from the deficits caused by the damage to V1 more than just as a relay point of visual information.

In both naive monkeys and humans, sustained activity during the delay period in the MGS task has been observed mainly in the cerebral regions such as the prefrontal and the parietal cortices (Gnadt and Andersen, 1988; Funahashi et al., 1989; Chafee and Goldman-Rakic, 1998; Brown et al., 2004; Srimal and Curtis, 2008). Based on reports that visually driven activity in the cerebral cortex was considerably attenuated by a lesion in V1 (Rodman et al., 1989; Schoenfeld et al., 2002; Schmid et al., 2009), the cerebral cortex, including the prefrontal and the parietal regions, would not be able to function exactly the same as it did before the lesion. Thus, our results seem well suited for the idea that the SC compensates for the role of the cerebral cortex in the retention of spatial memory after a V1 lesion.

Previous studies using naive monkeys reported that a part of the neurons in the SC also exhibits sustained activities in the absence of visual stimuli (Mays and Sparks, 1980; Kojima et al., 1996; Wurtz et al., 2001) and that these activities could be enhanced by the task condition (Ikeda and Hikosaka, 2007). Thus, the sustained activity in the ipsi SC might be achieved by the neural mechanism common to the intact brain. It is well known that the SC receives tonic inhibitory inputs from the substantia nigra pars reticulata (Hikosaka and Wurtz, 1983; Hikosaka et al., 2000), and the release from this tonic inhibition is proposed as a neural mechanism for modulation of the neuronal activity in the SC of the naive monkeys (Ikeda and Hikosaka, 2003). The same mechanism might help generate the sustained activity after a V1 lesion. However, such modulatory effects on the delay period activity in naive monkeys were observed only in a small fraction of the neurons with saccadic bursts, while the majority of the neurons in the ipsi SC showed sustained activities during the delay period regardless of the presence or absence of saccadic bursts. Therefore, it seems likely that a kind of plastic changes in response to the V1 lesion (Leh et al., 2006; Bridge et al., 2008; Nelles et al., 2009) underlies the sustained activity in the ipsi SC. Given that a previous study reported that visually driven activity was absent in the deeper layer of the SC after lesion and during inactivation of the V1 in anesthetized, untrained monkeys (Schiller et al., 1974), the visual response observed in this study seems to emerge as a result of the recovery processes and the behavioral training. Thus, the presence of the visual response also suggests the plastic changes around the SC.

It is interesting to note that the neurons in the ipsi SC exhibited an increase in their firing rates before the onset of the stimuli, as if to boost the visual response. This activity may reflect the expectation of a target or cue appearance in the affected visual field because the target or cue was presented in the affected or normal visual field in blocked trials, and might represent a compensatory mechanism for visually driven activity in the ipsi SC. The prestimulus activity seems to not directly contribute to the monkeys' performance; the magnitudes of the prestimulus activity (mean firing rates in the $100 \mathrm{~ms}$ period just before the cue onset) were not significantly different between the correct and error trials in the MGS task (Mann-Whitney $U$ test, $p>0.3, n=25$ ).

The SC, especially the deeper layer of the SC, is known to have direct and indirect reciprocal connections between the prefrontal and parietal cortex, such as the frontal eye field (FEF) and the lateral intraparietal area (LIP) (Leichnetz et al., 1981; Lynch et al., 1985, 1994; Paré and Wurtz, 1997; Sommer and Wurtz, 1998, Wurtz et al., 2001). Signal transmission between the SC and these cerebral regions might be facilitated by plastic changes after damage to V1, and the sustained activity might be maintained through this reciprocal network involving the SC, FEF, and LIP. Further study is needed to clarify the role of the prefrontal and the parietal regions in the residual spatial memory after damage to $\mathrm{V} 1$.

Monkeys with a unilateral lesion of V1 have been used as an animal model of blindsight (Cowey and Stoerig, 1995; Moore et al., 1995). Monkeys with a V1 lesion behave as if they are not aware of visual stimuli in the affected visual field when they have to report the presence or absence of targets, similarly as patients with blindsight. As in the previous studies, we have observed that the same subjects as those in the current study failed to detect visual stimuli in the affected visual field in the task to report the presence or absence of targets with saccades (M. Yoshida, K. Takaura, and T. Isa, manuscript in preparation). Thus, our results suggest that spatial information can be retained under the condition of blindsight. The ability to retain information for several seconds to guide goal-directed behaviors is often called "working memory" (Baddeley and Hitch, 1974), and its relationship with visual awareness has been discussed on many occasions (Baars, 1988; Crick and Koch, 1995; Dehaene and Naccache, 2001; Engel and Singer, 2001; Baars and Franklin, 2003), some of which proposed that working memory and visual awareness would be tightly linked to each other (Baars, 2003; Koch, 2004). Although here we examined only spatial memory for saccades, our results can be a counter-evidence to this proposal and might challenge the view on the relationship between visual awareness and working memory.

\section{References}

Baars BJ (1988) A cognitive theory of consciousness. Cambridge, UK: Cambridge UP.

Baars BJ (2003) Working memory requires conscious processes, not vice versa: a global workspace account. In: Neural basis of consciousness (Osaka N, ed), pp11-26. Amsterdam: Benjamins.

Baars BJ, Franklin S (2003) How conscious experience and working memory interact. Trends Cogn Sci 7:166-172.

Baddeley AD, Hitch GJL (1974) Working memory. In: The psychology of learning and motivation advances in research and theory (Bower GH, ed), pp 47-89. New York: Academic.

Boehnke SE, Munoz DP (2008) On the importance of the transient visual response in the superior colliculus. Curr Opin Neurobiol 18:544-551.

Boyer JL, Harrison S, Ro T (2005) Unconscious processing of orientation and color without primary visual cortex. Proc Natl Acad Sci U S A 102:16875-16879.

Bridge H, Thomas O, Jbabdi S, Cowey A (2008) Changes in connectivity after visual cortical brain damage underlie altered visual function. Brain 131:1433-1444. 
Brown MRG, DeSouza JFX, Goltz HC, Ford K, Menon RS, Goodale MA, Everling S (2004) Comparison of memory- and visually guided saccades using event-related fMRI. J Neurophysiol 91:873-889.

Campion J, Latto R, Smith YM (1983) Is blindsight an effect of scattered light, spared cortex and near-threshold vision? Behav Brain Sci 6:423-448.

Chafee MV, Goldman-Rakic PS (1998) Matching patterns of activity in primate prefrontal area $8 \mathrm{a}$ and parietal area 7ip neurons during a spatial working memory task. J Neurophysiol 79:2919-2940.

Cowey A, Stoerig P (1995) Blindsight in monkeys. Nature 373:247-249.

Crick F, Koch C (1995) Are we aware of neural activity in primary visual cortex? Nature 375:121-123.

Curtis CE, D'Esposito M (2003) Persistent activity in the prefrontal cortex during working memory. Trends Cogn Sci 7:415-423.

Daniel PM, Whitteridge D (1961) The representation of the visual field on the cerebral cortex in monkeys. J Physiol 159:203-221.

Dehaene S, Naccache L (2001) Towards a cognitive neuroscience of consciousness: basic evidence and a workspace framework. Cognition 79:1-37.

Dineen J, Hendrickson A, Keating EG (1982) Alterations of retinal inputs following striate cortex removal in adult monkey. Exp Brain Res 47:446-456.

Engel AK, Singer W (2001) Temporal binding and the neural correlates of sensory awareness. Trends Cogn Sci 5:16-25.

Funahashi S, Bruce CJ, Goldman-Rakic PS (1989) Mnemonic coding of visual space in the monkey's dorsolateral prefrontal cortex. J Neurophysiol 61:331-349.

Gnadt JW, Andersen RA (1988) Memory related motor planning activity in posterior parietal cortex of macaque. Exp Brain Res 70:216-220.

Gross CG, Moore T, Rodman HR (2004) Visually guided behavior after V1 lesions in young and adult monkeys and its relation to blindsight in humans. Prog Brain Res 144:279-294.

Hikosaka O, Wurtz RH (1983) Visual and oculomotor functions of monkey substantia nigra pars reticulate III. Memory-contingent visual and saccade responses. J Neurophysiol 49:1268-1284.

Hikosaka O, Takikawa Y, Kawagoe R (2000) Role of the basal ganglia in the control of purposive saccadic eye movements. Physiol Rev 80:953-978.

Ikeda T, Hikosaka O (2003) Reward-dependent gain and bias of visual responses in primate superior colliculus. Neuron 39:693-700.

Ikeda T, Hikosaka O (2007) Positive and negative modulation of motor response in primate superior colliculus by reward expectation. J Neurophyisol 98:3163-3170.

Ikeda T, Yoshida M, Isa T (2011) Lesion of primary visual cortex in monkey impairs the inhibitory but not the facilitatory cueing effect on saccade. J Cogn Neurosci 23:1160-1169.

Isa T, Yoshida M (2009) Saccade control after V1 lesion revisited. Curr Opin Neurobiol 19:608-614.

Koch C (2004) The quest for consciousness: a neurobiological approach. Englewood, CO: Roberts.

Kojima J, Matsumura M, Togawa M, Hikosaka O (1996) Tonic activity during visuo-oculomotor behavior in the monkey superior colliculus. Neurosci Res 26:17-28.

Leh SE, Johansen-Berg H, Ptito A (2006) Unconscious vision: new insight into the neuronal correlate of blindsight using diffusion tractography. Brain 129:1822-1832.

Leh SE, Ptito A, Schönwiesner M, Chakravarty MM, Mullen KT (2010) Blindsight mediated by an S-cone-independent collicular pathway: an fMRI study in hemispherectomized subjects. J Cogn Neurosci 22: 670-682.

Leichnetz GR, Spencera RF, Hardya SGP, Astruca J (1981) The prefrontal corticotectal projection in the monkey; an anterograde and retrograde horseradish peroxidase study. Neuroscience 6:1023-1041.

Lynch JC, Graybiel AM, Lobeck LJ (1985) The differential projection of two cytoarchitectonic subregions of the inferior parietal lobule of macaque upon the deep layers of the superior colliculus. J Comp Neurol 235:241-254.

Lynch JC, Hoover JE, Strick PL (1994) Input to the primate frontal eye field from the substantia nigra, superior colliculus, and dentate nucleus demonstrated by transneuronal transport. Exp Brain Res 100:181-186.
Mays LE, Sparks DL (1980) Dissociation of visual and saccade-related responses in superior colliculus neurons. J Neurophysiol 43:207-232.

Mohler CW, Wurtz RH (1977) Role of striate cortex and superior colliculus in visual guidance of saccadic eye movements in monkeys. J Neurophysiol 40:74-94.

Moore T, Rodman HR, Repp AB, Gross CG (1995) Localization of visual stimuli after striate cortex damage in monkeys: parallels with human blindsight. Proc Natl Acad Sci U S A 92:8215-8218.

Morris JS, Ohman A, Dolan RJ (1999) A subcortical pathway to the right amygdala mediating "unseen" fear. Proc Natl Acad Sci U S A 96: 1680-1685.

Morris JS, DeGelder B, Weiskrantz L, Dolan RJ (2001) Differential extrageniculostriate and amygdala responses to presentation of emotional faces in a cortically blind field. Brain 124:1241-1252.

Nelles G, Pscherer A, de Greiff A, Forsting M, Gerhard H, Esser J, Diener HC (2009) Eye-movement training-induced plasticity in patients with poststroke hemianopia. J Neurol 256:726-733.

Paré M, Wurtz RH (1997) Monkey posterior parietal cortex neurons antidromically activated from superior colliculus. J Neurophysiol 78: 3493-3497.

Pöppel E, Held R, Frost D (1973) Letter: residual visual function after brain wounds involving the central visual pathways in man. Nature 243:295-296.

Quintana J, Fuster JM (1999) From perception to action: Temporal integrative functions of prefrontal and parietal neurons. Cereb Cortex 9:213-221.

Rainer G, Rao SC, Miller EK (1999) Prospective coding for objects in primate prefrontal cortex. J Neurosci 19:5493-5505.

Ro T, Shelton D, Lee OL, Chang E (2004) Extrageniculate mediation of unconscious vision in transcranial magnetic stimulation-induced blindsight. Proc Natl Acad Sci U S A 101:9933-9935.

Rodman HR, Gross CG, Albright TD (1989) Afferent basis of visual response properties in area MT of the macaque. I. Effects of striate cortex removal. J Neurosci 9:2033-2050.

Sanders MD, Warrington EK, Marshall J, Wieskrantz L (1974) "Blindsight": vision in a field defect. Lancet 1:707-708.

Schiller PH, Stryker M, Cynader M, Berman N (1974) Response characteristics of single cells in the monkey superior colliculus following ablation or cooling of visual cortex. J Neurophysiol 37:181-194.

Schmid MC, Panagiotaropoulos T, Augath MA, Logothetis NK, Smirnakis SM (2009) Visually driven activation in macaque areas V2 and V3 without input from the primary visual cortex. PLoS One 4:e5527.

Schmid MC, Mrowka SW, Turchi J, Saunders RC, Wilke M, Peters AJ, Ye FQ, Leopold DA (2010) Blindsight depends on the lateral geniculate nucleus. Nature 466:373-377.

Schoenfeld MA, Noesselt T, Poggel D, Tempelmann C, Hopf JM, Woldorff MG, Heinze HJ, Hillyard SA (2002) Analysis of pathways mediating preserved vision after striate cortex lesions. Ann Neurol 52:814-824.

Segraves MA, Goldberg ME, Deng SY, Bruce CJ, Ungerleider LG, Mishkin M (1987) The role of striate cortex in the guidance of eye movements in the monkey. J Neurosci 7:3040-3058.

Sommer MA, Wurtz RH (1998) Frontal eye field neurons orthodromically activated from the superior colliculus. J Neurophysiol 80:3331-3335.

Srimal R, Curtis CE (2008) Persistent neural activity during the maintenance of spatial position in working memory. Neuroimage 39:455-468.

Van Essen DC, Newsome WT, Maunsell JH (1984) The visual field representation in striate cortex of the macaque monkey: asymmetries, anisotropies, and individual variability. Vision Res 24:429-448.

Weiskrantz L (1986) Blindsight: A case study and implications. Oxford: Oxford UP.

Weiskrantz L, Warrington EK, Sanders MD, Marshall J (1974) Visual capacity in the hemianopic field following a restricted occipital ablation. Brain 97:709-728.

Wurtz RH, Sommer MA, Paré M, Ferraina S (2001) Signal transformations from cerebral cortex to superior colliculus for the generation of saccades. Vision Res 41:3399-3412.

Yoshida M, Takaura K, Kato R, Ikeda T, Isa T (2008) Striate cortical lesions affected deliberates decision and control of saccade: Implication for blindsight. J Neurosci 28:10517-10530. 